\title{
Intensive cooling of large surfaces with arrays of jets
}

\author{
by Carosena Meola, Giovanni Maria Carlomagno \\ Department of Energetics Thermofluidynamics and Environmental Control (DETEC) \\ University of Naples Federico II, P.le Tecchio 80, 80125 Napoli, ITALY
}

\begin{abstract}
The attention of the present paper is focused on the intensive heat removal with arrays of impinging jets. Three jet configurations are tested which involve variation of geometric parameters such as nozzle shape, diameter and nozzle-tonozzle spacing and impingement distance and of flow rate. The convective heat transfer coefficient is measured with infrared thermography applied to the heatedthin-foil technique. Data are reduced in dimensionless form in terms of average Nusselt number and plotted against the Reynolds number and the impingement distance. The average Nusselt number values are also expressed as a function of the Reynolds number following the correlations available.
\end{abstract}

\section{Introduction}

The intensive removal of heat from large surfaces is a crucial task in many industrial processes amongst others the annealing of metals and plastic sheets and the tempering of glass. In fact, rapid and uniform cooling is vital for steel to reach, at the same time in the whole surface, the martensite transformation. This allows increase of hardness in depth, minimization of surface cracking and distortion; thus, the mechanical properties improve resulting in a longer life.

Starting after the Second World War and especially between the sixties and seventies, within the industrial boom, there has been an increasing demand of cooling devices. Jets of fluid were chosen for the advantages of high heat and/or mass transfer rates that occur in the impingement region. The heat transfer may be influenced by many factors involving both geometrical (nozzle geometry, nozzle-tonozzle spacing, impingement distance, etc.) and fluid flow conditions (turbulence, entrainment, jet velocity, spent air exhaust, etc.)

Therefore, the design of jets cooling devices with high ratio enhanced efficiency over power costs has been a challenge for many researchers and technicians during the past fifty years and is still an open question. The experimental tests, needed for the validation of such devices, have provided a multitude of data. In the same time, the interest of many researchers was devoted to gain insights into the fluid dynamics of impinging jets. As a consequence, literature is full of papers (theoretical, experimental and numerical) concerned with either a single, or an array of impinging jets, aimed at going through specific aspects such as the flow pattern after impingement [1], entrainment effects [2], unsteady effects [3], influence of nozzle shape [4], influence of shear layer dynamics [5].

In real applications, arrays of jets are used; a multi-jet system is endowed with interactions of adjacent jets that do not occur in the case of a single jet [6]. The interference between adjacent jets prior impingement is enhanced for closely spaced jets and large impingement distances; on the contrary, if the impingement distance is small, interactions due to collision of surface flows associated with the adjacent impinging jets become important. Data present in literature refer mainly to specific 
practical applications, which involve round nozzles [7] or jet orifice plates [8], confined jets with spent air exhausted between adjacent jets [9]. Measurements were also performed with different techniques (liquid crystals, thermocouples, etc.) each of them having a different precision which makes difficult a data generalization.

The experimental data, available for multi-jet systems, are mainly for relatively low values of the Reynolds number (i.e, $R e \leq 30000$ ). Little attention has been devoted to $R e$ values in the range $30000-60000$ and in rare exceptions $R e$ exceeds 70000 . On the contrary, in many industrial applications intensive heat removal is required, which also involves high mass flow rates and high $R e$ values. However, experimental tests are time consuming for the industry, while the simulation in laboratory of a multi-jet system working at high flow rate is rather expensive and also poses problems related to the Safety \& Health at work Regulations.

In the present paper we report some results obtained with multi-jet systems designed for intensive heat removal. The main objective is to add information about heat transfer for $R e$ in the range from 30000 to 80000 . The obtained data are also reduced following the available empirical equations to gain new insights for a more general data correlation.

\section{Experimental measurements}

\subsection{Test apparatus}

The experimental apparatus is sketched in figure 1. A constantan foil, which is $200 \mathrm{~mm}$ wide, $470 \mathrm{~mm}$ long and $0.050 \mathrm{~mm}$ thick, simulates the target surface. The target surface is held flat by a stiffening fixture and is heated by Joule effect, the electric current provided with a direct-current power supply within $1 \%$ uncertainty. The cooling air, supplied by a blower, goes through a pressure regulating valve, a heat exchanger and then to a plenum chamber where pressure and temperature are metered and flows through the array of nozzles.

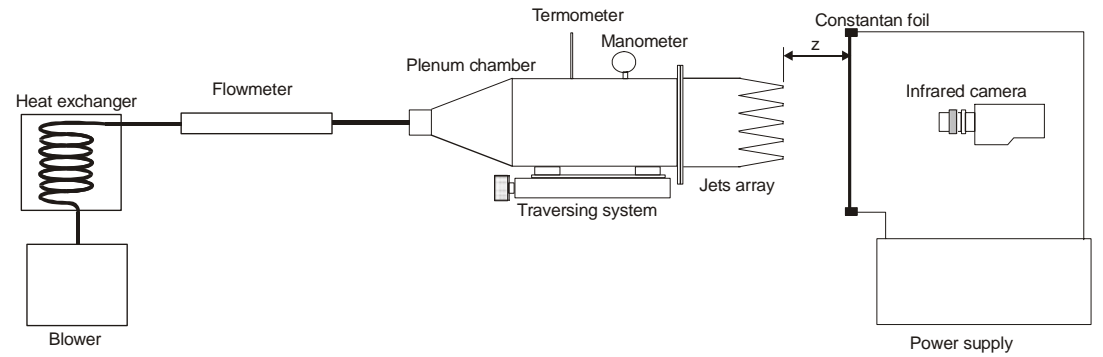

Fig. 1. Test apparatus

Three staggered arrays of jets, which are simply named $A f, A g$ and $A s$, are tested that involve variation of:

Geometry - includes variation of nozzles shape, diameter $D$ and spacing $x_{x}$ and $x_{y}$ along the $x$ and the $y$ directions respectively. Therefore, the nozzle-to-nozzle spacing is taken into account through two parameters: $x_{n}=\sqrt{x_{x} \cdot x_{y}}$ and $x_{r}=x_{x} / x_{y} . A g$ includes 5 blades with $5 \times 5$ round nozzles of $D=7.5 \mathrm{~mm}, x_{n}=30$, and $x_{r}=1.5$. As includes 5 blades with $5 \times 5$ rectangular nozzles of equivalent diameter $D=8.2 \mathrm{~mm}$, 
$x_{n}=32$ and $x_{r}=0.85$. Af includes 3 blades each with 9 staggered round nozzles of $D=7.5 \mathrm{~mm}, x_{n}=30$ and $x_{r}=1.14$.

Flow rate - is varied to have the Reynolds number, based on the nozzle diameter, in the range $30000-80000$.

Impingement distance - $z$ is varied from 4 up to 8.3 diameters.

The choice of round and rectangular nozzles is made not only for a merely variation of test parameters, but mainly to gain insights into the role played by the nozzles geometry on heat transfer. In fact, rectangular nozzles, arranged on blades, offer some advantages over circular nozzles mainly owing to the spent air exhaust. The nozzles spacing along $x$ and $y$ is also varied to have $x_{r}>1$, or $x_{r}<1$.

\subsection{Test procedure and instrumentation}

The convective heat transfer coefficient, $h$, is calculated from the relationship:

$$
h=\frac{\dot{q}-\dot{q}_{l}}{T_{w}-T_{a w}}
$$

with $\dot{q}$ the Joule heating (of the order of $5 \mathrm{~kW} / \mathrm{m}^{2}$ ), $\dot{q}_{l}$ the losses which are due to radiation, conduction and natural convection, $T_{w}$ the wall temperature and $T_{a w}$ the adiabatic wall temperature. $T_{w}$ and $T_{a w}$ are measured by infrared thermography applied to the heated-thin-foil technique [5]. The used infrared system is the Agema Thermovision 900 LW equipped with a MCT detector sensing the infrared energy in the 8-12 $\mu \mathrm{m}$ wavelength band. The surface of the constantan target plate is covered, over the side viewed by the infrared camera, with a thin film of opaque paint of emissivity $\varepsilon=0.95$. The emissivity is measured with the infrared system itself.

The test procedure is the following: switch on the blower, reach the desired flow rate value; wait (about 2 hours) to reach steady thermal conditions; then visualise the adiabatic wall temperature $T_{a w}$ and acquire the cold image; read the mercury thermometer for the temperature in the plenum chamber $T_{O}$ while recording the image for $T_{a w}$; put on the electric power supply; wait about 15 minutes to reach steady thermal conditions, visualise the wall temperature $T_{w}$ and acquire the corresponding thermographic image (hot image); read again the mercury thermometer for $T_{0}$ while recording the image for $T_{w}$. The temperature in the plenum chamber is checked to assure that both cold $\left(T_{a w}\right)$ and hot $\left(T_{w}\right)$ images are acquired at the same $T_{O}$ value (generally very small variations occur between the two acquisitions). Each data set is obtained by averaging 32 thermal images in time sequence to reduce measurement noise.

\section{Data analysis}

The acquired images are analysed and the $h$ values (Eq.1) are evaluated. Losses due to radiation $\dot{q}_{r}$ are evaluated according to the radiosity relation that expresses heat transfer by radiation between two grey bodies, one having a larger surface compared to the other: 


$$
\dot{q}_{r}=\varepsilon \sigma\left(T_{w}^{4}-T_{a}^{4}\right)
$$

where $\varepsilon$ is the total emissivity coefficient, $\sigma$ is the Stefan-Boltzmann constant and $T_{a}$ is the ambient temperature. The tangential conduction $\dot{q}_{c}$ is evaluated by means of the second derivative of the wall temperature $T_{w}$ :

$$
\dot{q}_{c}=s k_{f}\left(\frac{\partial^{2} T_{w}}{\partial x^{2}}+\frac{\partial^{2} T_{w}}{\partial y^{2}}\right)
$$

where $s$ is the thickness, $k_{f}$ the thermal conductivity coefficient of the foil and $x$ and $y$ the coordinates parallel to the foil surface. Losses due to natural convection are neglected. A typical 3D map of $h$, for $A g$, impingement distance of 4 diameters and effective flow rate $9 \mathrm{~kg} / \mathrm{sm}^{2}$, is shown in figure 2 .

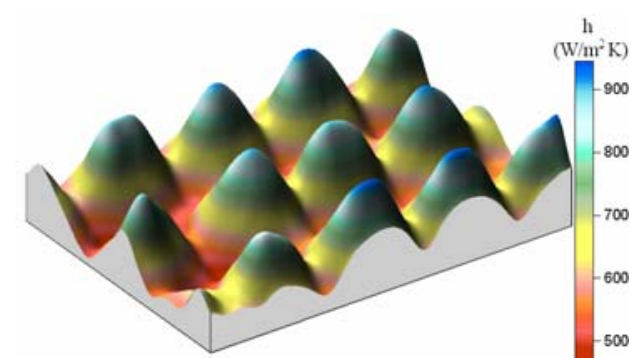

Fig. 2. $3 D$ h map for round nozzles $(A g)$ at $z / D=4$ and $\dot{m}_{\text {eff }}=9 \mathrm{~kg} / \mathrm{m}^{2} s$

The effective flow rate is calculated as ratio between the measured flow rate $\dot{m}$ and the total open area $A_{f}$ :

$$
\dot{m}_{\text {eff }}=\frac{\dot{m}}{A_{f}}=\frac{\dot{m}}{n x_{x} x_{y}}
$$

where $n$ is the number of nozzles and $x_{x}$ and $x_{y}$, as already specified, are the distances between nozzles in $x$ and $y$ directions respectively. As expected, the heat transfer attains a maximum in correspondence of each jet centre and laterally decreases towards minima between adjacent jets.

\subsection{Dimensionless data reduction}

Data are reduced in dimensionless form in terms of the average Nusselt number $\overline{N u}$ defined as:

$$
\overline{N u}=\frac{\bar{h} D}{k_{a}}
$$


$\bar{h}$ is the convective heat transfer coefficient averaged over the area of the hexagon (figure 3 ), $k_{a}$ is the conductivity coefficient of air evaluated at the film temperature $T_{f}$ :

$$
T_{f}=\frac{T_{w}+T_{a w}}{2}
$$

Of course, $k_{a}$ is determined point by point on the whole imaged surface.

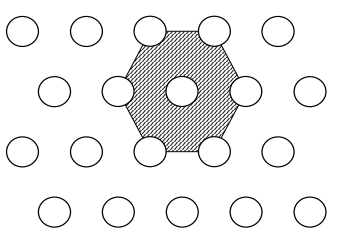

Fig. 3. Sketch of averaging area

The $\overline{N u}$ values for the three configurations $A f, A g$ and $A s$ are plotted against $z / D$ in figure $4(\mathrm{a}, \mathrm{b}, \mathrm{c})$. As expected, $\overline{N u}$ increases with increasing $R e$ and with decreasing $z / D$; on the overall, the lowest $\overline{N u}$ values are obtained with the configuration $A f$ and the highest $\overline{N u}$ values with As. A comparison between the three tested jet configurations is shown in figure 5 where the $\overline{N u}$ values, for $z=40 \mathrm{~mm}$, are plotted against $R e$. As can be seen, the data for the three configurations lie over three lines, which are almost parallel. It has to be considered that $z / D$ takes the value of 4.87 for $A s$ and of 5.3 for both $A f$ and $A g$. However, this has little influence because the two lines for $A g$ and $A s$ are very close, while a large distance separates the two lines across data by $A f$ and $A g$ that are for the same $z / D$. What plays a fundamental role is the arrangement of nozzles over blades. Most probably, in the case of $A f$, for which two nozzles lines lie over the same blade, the spent air interferes with the oncoming jet lowering the heat transfer.

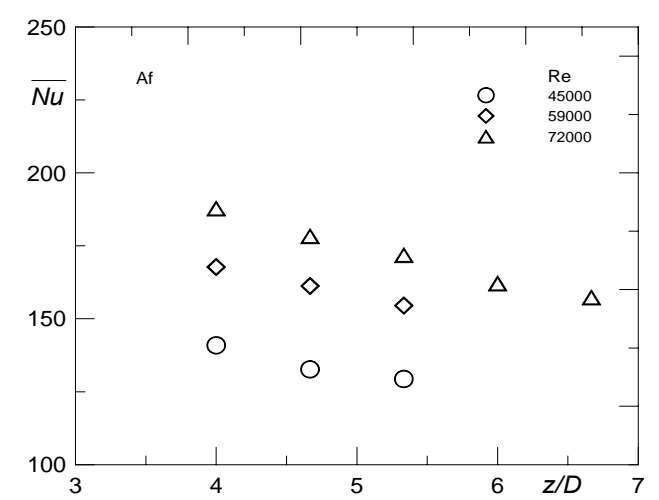

a) $A f$ 
http://dx.doi.org/10.21611/qirt.2006.064

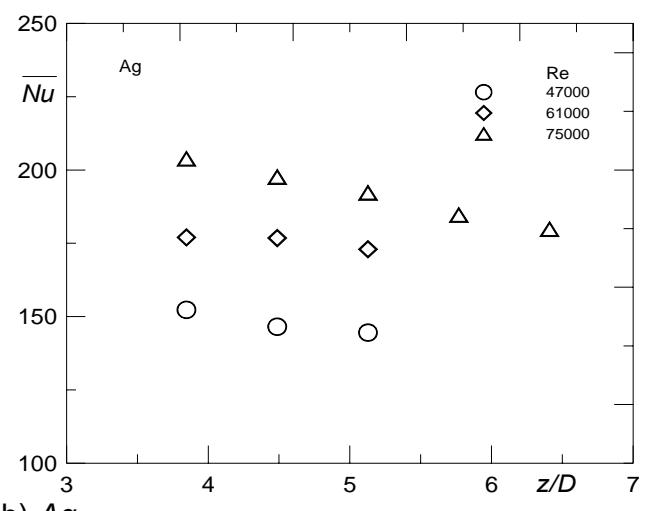

b) $\mathrm{Ag}$

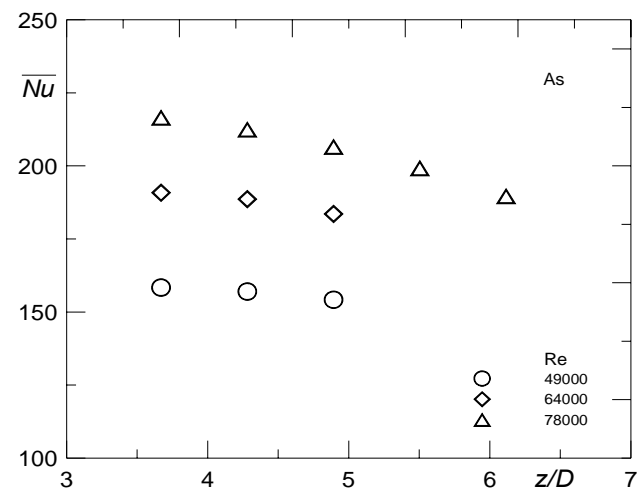

c) As

Fig. 4. Distribution of $\overline{N u}$ with varying $R e$ and $z / D$ for the tested jet arrays

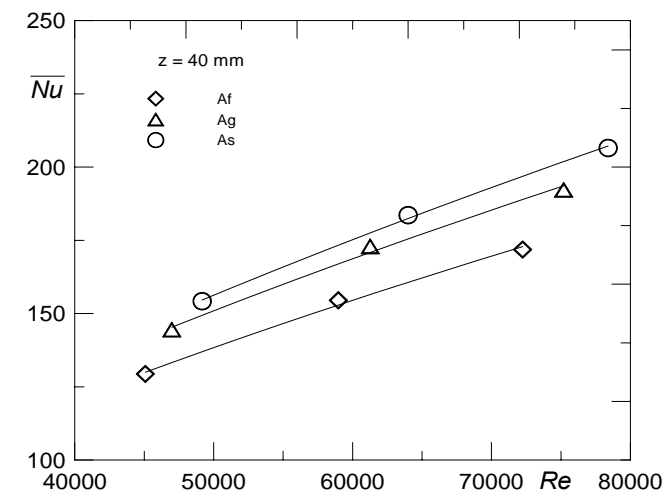

Fig. 5. A comparison between the tested jet arrays for $z=40 \mathrm{~mm}$ 


\section{Data correlation}

To gain information useful for a general data correlation $\overline{N u}$ values are reduced following three different correlations. One correlation, proposed by Gardon and Cobonpue [7]:

$$
\overline{N u}_{s}=0.286 R e_{a}^{0.625}
$$

$\overline{N u}_{s}$ is the Nusselt number based on the nozzle spacing $x_{n}$ (for a square array):

$$
\overline{N u}_{s}=\frac{\bar{h} x_{n}}{k_{a}}
$$

The Reynolds number $R e_{a}$, based on the arrival velocity $u_{a}[10]$ and $x_{n}$ :

$$
\operatorname{Re}_{a}=\frac{u_{a} x_{n}}{v}
$$

being $v$ the kinematic viscosity. The experimental data obtained during the present investigation are reduced according to Eqs.(8) and (9) and shown in figure 6 together with the plot of Eq.(7). As can be seen the points that refer to the array As with rectangular nozzles display the highest spread; this is likely due to the fact that Eq. (7) was obtained with a square array of round nozzles. Indeed, the relationship between Nusselt and Reynolds numbers depends on many test parameters which are: the geometry of the array (round nozzles, slots, nozzle-to-nozzle distance, etc.) of jets, the impingement distance $(z / D)$, the characteristics of the employed fluid (viscosity, specific heat, etc.) and so forth.

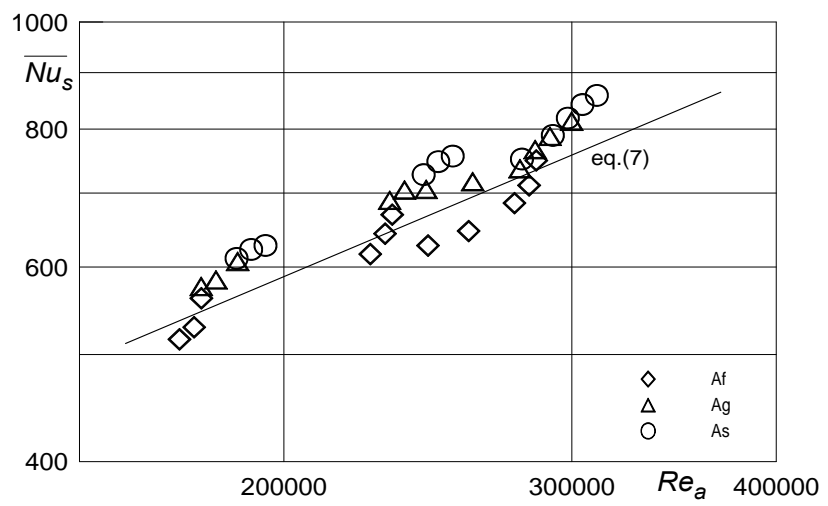

Fig. 6. Present data correlated according to Eq. (7)

Another equation is that proposed by Martin [11] for an array of round nozzles:

$$
\frac{\overline{N u}}{\operatorname{Pr}^{0.42} K(z / D, f) G(z / D, f)}=F(R e)
$$


$\mathrm{Nu}$ and $R e$ are calculated in the classical way (nozzles diameter $D$ and exit velocity $u), f$ is the ratio of nozzle exit cross section to the area $A$ of the square, or the hexagon, attached to it:

$$
\begin{aligned}
& f=\frac{\frac{\pi}{4} D^{2}}{A} \\
& K(z / D, f)=\left[1+\left(\frac{z / D}{0.6 / \sqrt{f}}\right)^{6}\right]^{-0.05}
\end{aligned}
$$

and

$$
G(z / D, f)=2 \sqrt{f} \frac{1-2.2 \sqrt{f}}{1+0.2(z / D-6) \sqrt{f}}
$$

the range of validity for Eqs.(12) and (13) is:

$$
\begin{aligned}
& 2000 \leq R e \leq 100000 \\
& 0.004 \leq f \leq 0.04 \\
& 2 \leq z / D \leq 12
\end{aligned}
$$

the second term in Eq.(10) is:

$$
F(R e)=0.5 R e^{2 / 3}
$$

in the range of values indicated by the first line of Eq.(14). For the tested configurations we have:

$$
\begin{aligned}
& 30000 \leq R e \leq 80000 \\
& f=0.05 \\
& 4 \leq z / D \leq 8.3
\end{aligned}
$$

and thus $f$ is outside the range of validity of Eq.(14). Notwithstanding this, we try to apply the Martin correlation to our data. Therefore, data obtained in the present investigation are reduced, following Eq.(10), in terms of average Nusselt number divided the Prandtl number to a 0.42 power and the two functions Eqs.(12) and (13) and are plotted against $R e$ in figure 7 together with the plot of Eq.(15).

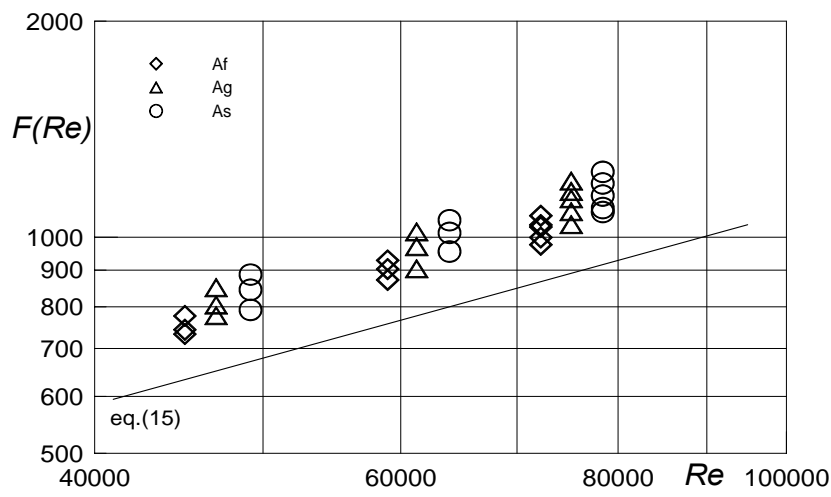

Fig. 7. Present data correlated according to Eqs. (10) and (15) 
As can be seen, present data lies over a curve which is about $25 \%$ higher than that resulting from the plot of Eq.(15). This might be ascribed to the over limiting value of $f$. Indeed, it is possible to see that, in figure 12b of the Martin survey [11], data of Glaser (1963) and data of Ott (1961) are respectively about $40 \%$ and about $20 \%$ higher with respect to the curve representing Eq.(15).

A third correlation can be obtained by replacing the first term in Eq.(10) with $\overline{N u}_{r}$ that is defined as:

$$
\overline{N u}_{r}=\frac{\overline{N u}}{\operatorname{Pr}^{0.42}\left(\frac{z / D}{\sqrt{f}}\right)^{-0.3}}
$$

$\overline{N u}_{r}$ is plotted against $R e$ in figure 8. It is found that, for the three jet array configurations, the experimental data are well fitted by the relationship:

$$
\overline{N u}_{r}=0.5 R e^{0.625}
$$

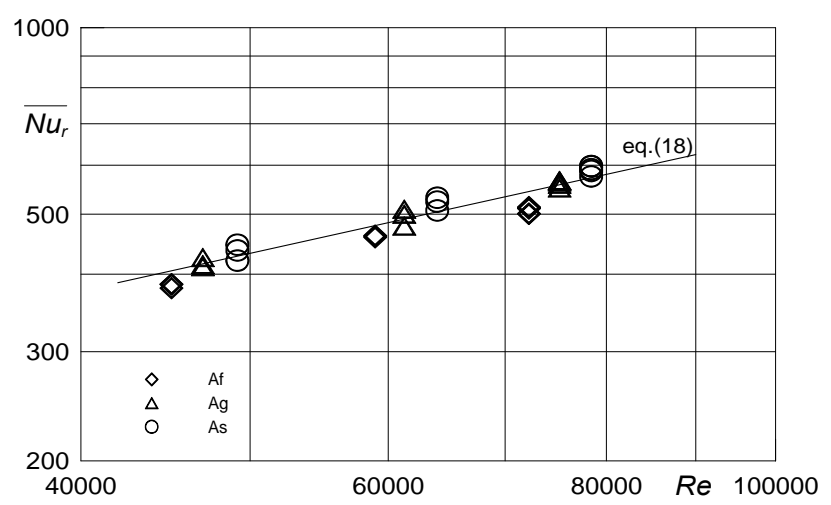

Fig. 8. Present data reduced according to Eqs. (17)

Owing to the fact that also Eq.(7) correlates quite well present data, a combination of Eq.(18) with Eq.(7) may be used to find a relationship between impingement distance and nozzles parameters (diameter, nozzle-to-nozzle spacing and relative area). In fact, by extracting $\bar{h} / k_{a}$ from both equations and equalling, it is found:

$$
0.286 \frac{1}{x_{n}}\left(\frac{u_{a} x_{n}}{v}\right)^{0.625}=0.5 \operatorname{Pr}^{0.42} \frac{1}{D}\left(\frac{z / D}{\sqrt{f}}\right)^{-0.3}\left(\frac{u D}{v}\right)^{0.625}
$$

and taking into account that for short impingement distances (inside the potential core region) $u_{a}=u$, Eq.(19) leads to:

$$
\frac{z}{D} \cong 4 \sqrt{f}\left(\frac{x_{n}}{D}\right)^{5 / 4}
$$


while in the fully developed region, being $u_{a}=6.63 u D / z$ [13], Eq.(19) leads to:

$$
\frac{z}{D} \cong 2.15 f^{-0.46}\left(\frac{x_{n}}{D}\right)^{7 / 6}
$$

and, being $f$ linked to $D$ and $x_{n}$ through Eq.(11), Eqs.(20) and (21) establish a direct relationship between $z / D$ and $x_{n} / D$.

\section{Conclusions}

The obtained results show that the highest heat transfer rates are achieved by rectangular nozzles. All data, regardless of the geometry of the nozzles array, are well correlated by Eq.(18) with the dependency of Nusselt on $f$ and $z / D$ expressed in a simple way through Eq.(17). It seems that present data, apart from those obtained with rectangular nozzles, are also quite well fitted by the correlation found by Gardon and Cobonpue [7]. Conversely, the correlation proposed by Martin [11] is not suitable to reduce present data. Most probably this is because present data are for intensive cooling, which was not previously investigated in depth.

Acknowledgements The authors wish to thank Dr. T. Astarita for developing the software used to reduce thermal images and Mr. G. Sicardi for setting up the experimental apparatus.

\section{REFERENCES}

[1] M.B. Glauert. The wall jet J. Fluid Mechanics, 1 (1956) 625-643.

[2] R.J. Goldstein, K.A. Sobolik and W.S. Seol. Effect of entrainment on the heat transfer to a heated circular air jet impinging on a flat surface. J. Heat Transfer, 112 (1990) 608-611.

[3] B.R. Monkewitz, D.W. Bechert, B. Barsikow and B. Lehmann. Self-excited oscillations and mixing in a heated round jet. J. Fluid Mechanics, 213 (1990) 611-639.

[4] C. Meola, L. de Luca and G.M. Carlomagno. Azimuthal instability in an impinging jet: adiabatic wall temperature distribution. Experiments in Fluids, 18 (1995) 303-310.

[5] C. Meola, L. de Luca and G.M. Carlomagno. Influence of shear layer dynamics on impingement heat transfer. Experimental Thermal and Fluid Science, 13 (1996) 29-37.

[6] R. Viskanta. Heat transfer to impinging isothermal gas and flames jets. Experimental Thermal and Fluid Science, 6 (1993) 111-134.

[7] R. Gardon and J. Cobonpue. Heat transfer between a flat plate and jets of air impinging on it in: Int. Developments in Heat Transfer New York, (1962) 454460.

[8] A.I. Behbahani and R.J. Goldstein. Local heat transfer to staggered arrays of impinging circular air jets J. Engineering for Power, 105 (1983) 354-360.

[9] A.M. Huber and R. Viskanta. Convective heat transfer to a confined impinging array of air jets with spent air exits, J. Heat Transfer, 116 (1994) 570-576.

[10] J.F. Taylor, H.L. Grimmett and E.W. Comings. Isothermal free jets of air mixing with air Chemical Engineering Progress, 47 (1951) 175-180.

[11] H. Martin. Heat and mass transfer between impinging gas jets and solid surfaces in Advances in Heat Transfer ed. J.P. Hartnett and Th.F. Irvine, Academic Press New York 13 (1977) 1-60. 